\title{
A Destructive New Disease of Citrus in China Caused by Cryptosporiopsis citricarpa sp. nov.
}

Li Zhu, Xinghong Wang, Feng Huang, Jinze Zhang, and Hongye Li, Institute of Biotechnology, Zhejiang University, Hangzhou, Zhejiang, 310029, China; Dekuan Ding, Citrus Institute of Chenggu County, Shaanxi Province, Chenggu, Shaanxi, 723200; and Kevin D. Hyde, Institute of Excellence in Fungal Research, School of Science, Mae Fah Luang University, Chiang Rai, 57100, Thailand; King Saud University, College of Science, Botany and Microbiology Department, Saudi Arabia

\begin{abstract}
Zhu, L., Wang, X., Huang, F., Zhang, J., Li, H., Ding, D., and Hyde, K. D. 2012. A destructive new disease of Citrus in China caused by Cryptosporiopsis citricarpa sp. nov. Plant Dis. 96:804-812.

Defoliation, dieback and mortality of Satsuma mandarin (Citrus unshiu), as well as kumquat (Fortunella margarita), in Chenggu County, Shaanxi Province, China was first noticed in 2006 and caused substantial economic losses to citrus production. The incidence of leaves infected approached $100 \%$ in some badly infected orchards. The disease prevailed only in late winter and early spring, where early symptoms were rounded and target-like spots on leaves. The disease was named target spot. Black conidiomata were observed on the upper surface of the leaves. A Cryptosporiopsis species was consistently recovered from the infected leaves, shoots, and branches. Koch's postulates were ful-

filled by inoculating the conidial suspension of Cryptosporiopsis sp. onto the leaves of Satsuma mandarin. Phylogenetic analysis based on $L S U$ sequence data indicated that this taxon clustered in Cryptosporiopsis (teleomorph: Neofabraea, Dermateaceae). Phylogenetic analysis based on ITS, SSU, and TUB indicated that the isolates of Cryptosporiopsis sp. constituted a distinct clade. Further study also demonstrated that this taxon was morphologically distinct from other species of Cryptosporiopsis, thus suggesting it might belong to an undescribed species. The name Cryptosporiopsis citricarpa sp. nov. is given to accommodate the fungal pathogen in this study.
\end{abstract}

Many leaf diseases of citrus caused by pathogenic fungi have been reported worldwide. They include Alternaria brown spot ( $\mathrm{Al}$ ternaria alternata Fr. (Keissler) pv. citri Solel), mancha foliar de los citricos (A. limicola Simmons \& Palm), anthracnose (Colletotrichum gloeosporioides (Penz.) Penz. \& Sacc.), lime anthracnose (C. acutatum J.H. Simmonds), scab (Elsinoë fawcettii Bitancourt \& Jenk.), areolate leaf spot (Thanatephorus cucumeris (A.B. Frank) Donk), black spot (Guignardia citricarpa Kiely), greasy spot (Mycollosphaerella citri Whiteside), melanose (Diaporthe citri F.A. Wolf), phaeoramularia leaf spot (Phaeoramularia angolensis (Car. \& Men.) P.M. Kirk), and Septoria spot (Septoria citri Pass.) (23), as well as pomelo tan spot (Phyllosticta citriasiana N.F. Wulandari, Crous \& Gruyter) (27) and Cryptosporiopsis spot (Cryptosporiopsis citri P.R. Johnst. \& Full.) (12). These diseases usually occur on young leaves of various Citrus species during the warm, humid seasons. An undescribed disease of citrus reported here occurred in late winter and early spring in Chenggu County, Shaanxi Province of China. The disease also infected petioles, shoots, twigs, branches, and standing trunks. The infected leaves defoliated abundantly in early spring, followed by dieback, branch blight, and eventually death of whole trees. Named target spot, this disease has become a concern to the local citrus industry in parts of China. Such disease was not reported before based on our best knowledge.

Neofabraea (Dermateaceae, Helotiales, Leotiomycetes, Ascomycota) was introduced by Jackson (11) for N. malicorticis H.S. Jacks, which caused apple anthracnose, and the genus has been

Corresponding author: Hongye Li,

E-mail: hyli@zju.edu.cn; hongye_lily@yahoo.com

* The $\boldsymbol{e}$-Xtra logo stands for "electronic extra" and indicates that Figures 1, 5 , and 6 appear in color in the online edition.

Accepted for publication 22 December 2011.

http://dx.doi.org/10.1094/PDIS-09-11-0775

(c) 2012 The American Phytopathological Society widely accepted $(1,25)$. Six species of Neofabraea have been described based on genetic analysis of ITS, SSU, and TUB sequences (6) or morphology combined with ITS and $L S U$ sequence data (4). $N$. krawtzewii (also known as $N$. populi) is responsible for bark lesions on poplar (Populus) trees (21), but does not infect apple (Malus) or pear (Pyrus) (8). N. malicorticis, N. alba, N. perennans, and Cryptosporiopsis kienholzii are associated with bark canker and bull's eye rot of postharvest apple and pear $(7,8,10,17)$. Among these four Neofabraea species, $N$. malicorticis and $N$. perennans are responsible for anthracnose canker and perennial canker of pome fruit trees, respectively (7). Leaf spots or defoliation on these host plants by Neofabraea spp. have not been reported, except that N. eucalypti could lead to Eucalyptus leaf spots (4).

Cryptosporiopsis species are anamorphic Pezicula and Neofabraea, two genera of the Helotiales $(1,6,25)$. Species of Cryptosporiopsis are characterized by having extremely variable conidiomata, from simple acervuli to multilocular, rostrate eustromata, and relatively large straight, ellipsoid to pyriform, conidia, with a protuberant scar (19). The presence of ellipsoid, pluriguttulate, non-septate macroconidia and much smaller microconidia are also characteristic (25). Many species of Cryptosporiopsis are endophytes; some species are known to produce antibacterial, fungicidal, and herbicidal secondary metabolites $(16,18)$; whereas some species are pathogens of woody plants, causing serious plant disease $(7,15,17)$.

In this study, we describe a new leaf spot disease of citrus in China, illustrate morphological and molecular-based phylogenetic characteristics of its causal agent, and describe the cause as a new fungal species Cryptosporiopsis citricarpa.

\section{Materials and Methods}

Prevalence and incidence of disease. Periodic surveys of disease development on citrus were conducted from December 2009 to May 2011 in three orchards in Chenggu County, Shaanxi Province, China. Citrus leaves with disease symptoms were photographed. The incidence of diseased leaves or seedlings on Satsuma mandarin and kumquat was assessed on 16 February 2011 at eight orchards and two nurseries. In each orchard, five trees were selected randomly. Four shoots were randomly located on each tree, 
and five leaves from each of the shoots were investigated. Leaves were recorded as infected if a single lesion was observed. Four seedlings from each of five sites selected randomly in each seedling nursery were also investigated. The seedlings were recorded as infected if any leaf or any part of the stem showed disease symptoms.

Isolation of the causal agent. Diseased leaves, shoots, twigs, and branches were sent by local extension staff to our laboratory for identification of the pathogen in 2010. Further sampling was also conducted by the authors in February 2011. Direct tissue isolation of causal agents was performed from leaves, shoots, and branches that were surface-sterilized with $75 \%$ ethanol for $30 \mathrm{~s}$, $1 \% \mathrm{NaOCl}$ for $1 \mathrm{~min}$, and then rinsed with sterile distilled water. Small sections $(1 \times 2 \mathrm{~mm})$ from the margins of diseased and healthy tissues were placed onto potato dextrose agar (PDA) amended with $50 \mu \mathrm{g} / \mathrm{ml}$ streptomycin (PDAS) in petri dishes. Alternatively, monosporic isolates were obtained when sporulation was present on the surface of the lesions using a modified method of Goh (9). Briefly, an individual leaf square $(5 \times 5 \mathrm{~mm})$ bearing acervuli was immersed in 300 to $500 \mu \mathrm{l}$ of sterile distilled water. Small drops of suspension were placed on a glass slide and examined under a dissecting microscope at $\times 20$ magnification. Drops that contained only a single spore were transferred to PDAS plates. The plates were incubated at $20^{\circ} \mathrm{C}$ for 3 to 14 days. A colony that grew on PDAS was transferred onto fresh PDAS. Pure single-spore cultures were then transferred onto PDA slants and stored at $4{ }^{\circ} \mathrm{C}$ for further study or at $-80^{\circ} \mathrm{C}$ for long-term storage in $25 \%$ glycerol. Strains SXL1, SXL6, and SXL10 (Table 1) were selected at random for further study.

Pathogenicity tests. The three strains selected for experimentation gave similar results, and the results for strain SXL6 were therefore only reported here. The inoculation experiment was conducted on 27 May and 11 December 2010 following a modified method reported by Yang et al. (29). Five Satsuma mandarin plants grown in 20-liter flower pots maintained in an open field on Huajiachi Campus, Zhejiang University, Hangzhou, Zhejiang, China were inoculated with each of the isolates. Five leaves (on one shoot) from each plant were inoculated with each isolate. Conidia, produced on 2-week-old PDA plates cultured at $20^{\circ} \mathrm{C}$ under 12-h alternating cycles of dark and fluorescent light (suspended $300 \mathrm{~mm}$ above), were collected by pouring $2 \mathrm{ml}$ of sterile distilled water onto a plate and scraping the cultures gently with a sterile glass rod. The suspension was filtered through two layers of cheesecloth, and its concentration was adjusted to $10^{6}$ conidia $/ \mathrm{ml}$ using a hemacytometer. Leaves (emerged in the current year) were surface-disinfested with $75 \%$ ethanol using cotton swabs, air-dried, and then slightly wounded (but not pierced) on the upper surface with an insect needle. The fresh conidial suspension was evenly brushed onto the wounded leaves with a small, soft writing brush. The inoculated shoots were covered with moistened polyethylene bags to maintain a high humidity for 3 days. For the noninoculated controls, leaves were wounded as described above and brushed with sterile water only. Disease symptom development was monitored at 1-month intervals up to 10 May 2011. Reisolation of the fungus from some diseased leaves was attempted following the method described above to fulfill Koch's postulate on 22 February
2011. Incidence was obtained using the formula: incidence $(\%)=$ (infected leaves/inoculated leaves) $\times 100 \%$.

To test whether strain SXL6 could infect fruits, mature, nonfungicide-treated Satsuma mandarin fruits were subjected to pathogenicity testing following the modified methods reported by Xiao and Rogers (28) and Cai et al. (3). Fruits were surface-disinfested for $10 \mathrm{~min}$ in $2 \%$ detergent, rinsed three times with sterile distilled water, and then air-dried on a clean bench. The fruits were then wounded with a sterile insect needle to a depth of $1 \mathrm{~mm}$, and a 5mm-diameter mycelium plug cut from a 14-day-old PDA culture was placed onto the wounded areas of each fruit. The inoculated fruits were placed in plastic trays on a layer of moist absorbent paper and then wrapped with another layer of polyethylene film and stored at $20^{\circ} \mathrm{C}$ for 14 days to facilitate fungal growth. The treated fruits were then transferred to $4^{\circ} \mathrm{C}$ to mimic the conditions for disease development in orchards. Observations of disease symptom development were conducted periodically for 2 months after inoculation. The experiment was conducted twice. Data were analyzed as above.

Morphology, colony characteristics, and optimum temperature requirements. Morphological characteristics of the fungal fruiting bodies produced in vivo (on infected leaves) in orchards and in vitro (in culture) were examined using differential interference contrast microscopy (Eclipse 80i, Nikon, Japan), and photographed using a Nikon digital camera (NIS-Elements F3.0, Nikon, Japan). Herbarium specimens were deposited in the culture collection of the Centraalbureau voor Schimmelcultures (CBS) in Utrecht, the Netherlands, and in the Institute of Biotechnology, Zhejiang University, China.

Three strains (SXL1, SXL6, and SXL10; Table 1) isolated from leaves with typical target-like spot symptoms were used to determine their morphology. An isolate of C. kienholzii (CLX4144, isolated from apple fruit) provided by Chang-Lin Xiao (Washington State University, USA) was included for comparison. Colony characteristics were evaluated on PDA, oatmeal agar (OA), and malt extract agar (MEA) in 55-mm-diameter petri dishes after 14 days of incubation at $20^{\circ} \mathrm{C}$; colony diameters were also measured. To determine the effect of temperature on mycelial growth, mycelial plugs ( $5 \mathrm{~mm}$ diameter) were removed with a sterile cork borer from the leading edges of 14-day-old PDA cultures, and then transferred onto PDA in 55-mm-diameter petri dishes following the method of Xiao and Rogers (28). The cultures were incubated respectively at $0^{\circ} \mathrm{C}$ (in an ice cube), $4,10,16,20,25,28,30$, and $35^{\circ} \mathrm{C}$. There were three replicates for each temperature. Colony diameters of each plate were measured 14 days postincubation. Cultures that did not grow after 14 days at 30 or $35^{\circ} \mathrm{C}$ were placed at $20^{\circ} \mathrm{C}$ for 14 days to determine whether the fungus could resume growth. The daily mycelial growth rate was calculated, and the database was analyzed by IBM SPSS 16.0 (New York). The entire experiment was conducted twice.

Amplification and sequencing of $L S U, I T S, S S U$, and TUB genes. Mycelia (50 to $200 \mathrm{mg}$ ) grown on PDA at $20^{\circ} \mathrm{C}$ were scraped away from the agar surface at 14 days postincubation and resuspended in $1 \mathrm{ml}$ of extraction buffer (2\% CTAB [hydroxyacetyl trimethyl ammonium bromide], 2\% PVP, $1.4 \mathrm{mM} \mathrm{NaCl}, 100$ $\mathrm{mM}$ Tris- $\mathrm{HCl}$ [pH 8.0], $20 \mathrm{mM}$ EDTA [pH 8.0], $2 \%$-mercap-

Table 1. Information of partial isolates of Cryptosporiopsis citricarpa in this study

\begin{tabular}{|c|c|c|c|c|c|c|c|c|}
\hline \multirow[b]{2}{*}{ Isolates $^{\mathbf{a}}$} & \multirow[b]{2}{*}{ Sites } & \multirow[b]{2}{*}{ Host } & \multirow{2}{*}{$\begin{array}{c}\text { Tissue } \\
\text { type }\end{array}$} & \multirow{2}{*}{$\begin{array}{l}\text { Collection } \\
\text { date }\end{array}$} & \multicolumn{4}{|c|}{ GenBank accession } \\
\hline & & & & & ITS & $L S U$ & $S S U$ & $T U B$ \\
\hline SXL1 = CBS 130530 & Yaozhuang, Baoshan & Citrus unshiu & Leaf & 27-Mar-2010 & JN601676 & & JN601684 & JN601692 \\
\hline SXL2 = CBS 130531 & Wulang, Wulang & C. unshiu & Leaf & 22-Feb-2011 & JN601677 & & JN601685 & JN601693 \\
\hline SXL3 = CBS 130532 & Wulang, Wulang & C. unshiu & Shoot & 22-Feb-2011 & JN601678 & & JN601686 & JN601694 \\
\hline SXL4 = CBS 130533 & Dongzha nursery garden & C. unshiu & Leaf & 22-Feb-2011 & JN601679 & & JN601687 & JN601695 \\
\hline SXL5 = CBS 130534 & Chencun, Baoshan & C. unshiu & Leaf & 22-Feb-2011 & JN601680 & & JN601688 & JN601696 \\
\hline SXL6 = CBS 130297 & Unclear site in Chenggu & C. unshiu & Leaf & 27-Mar-2010 & JN601681 & JN601700 & JN601689 & JN601697 \\
\hline SXL10 = CBS 130535 & Dongzha nursery garden & Fortunella margarita & Leaf & 27-Mar-2010 & JN601682 & & JN601690 & JN601698 \\
\hline SXL11 = CBS 130536 & Guanwangbao, Bowang & C. unshiu & Leaf & 7-Mar-2011 & JN601683 & & JN601691 & JN601699 \\
\hline
\end{tabular}

a Some isolates obtained in 2010 and 2011 were not listed in this table. 
toethanol) in a microcentrifuge tube containing $100 \mathrm{mg}$ of $0.5 \mathrm{~mm}$ ceramic beads, ground by Precellys machine (Bertin, Montigny le Bretonneux) for $30 \mathrm{~s} \times 2$ at 5,000 rpm. Total DNA was further extracted using modified method of CTAB (24). A 1,300- to 1,400bp segment of the large subunit ribosomal DNA $(L S U)$ gene was amplified with primers LR0R and LR5 (26) from one isolate (SXL6) and used to determine the placement of the family and genus. To determine the placement of Cryptosporiopsis species, an approximately 500-bp DNA fragment containing full length of the internal transcribed spacer 1 , the $5.8 \mathrm{~S}$ ribosomal DNA, and the internal transcribed spacer 2 (ITS), a 500- to 600-bp fragment of rDNA encoding part of the mitochondrial small subunit ribosomal DNA $(S S U)$, and an approximately 600-bp fragment of partial $\beta$ tubulin gene $(T U B)$ was amplified using primer pairs UN-UP18S42/UN-LO28S-22 (2), NMS1/NMS2 (13), and Bt-T2m-Up/BtLEV-Lo1 (6), respectively. The primer sequences were listed in Table 2. Eight isolates derived from leaves and shoots (Table 1) were included. The PCR reaction mixture $(50 \mu \mathrm{l})$ contained 0.5 $\mu \mathrm{M}$ of each primer, $0.2 \mathrm{mM}$ dNTPs, $2.5 \mathrm{mM} \mathrm{MgCl} 2,1$ to $10 \mathrm{ng}$ total DNA template, and 2.5 units of Taq DNA polymerase in 10× buffer. PCR was performed using Thermal Cycler (BIO-RAD, S1000, California) with a 3-min $96^{\circ} \mathrm{C}$ denaturation step followed by 40 cycles of $45 \mathrm{~s}$ denaturation at $96^{\circ} \mathrm{C}, 1 \mathrm{~min}$ annealing at $55^{\circ} \mathrm{C}$ for $L S U, 68^{\circ} \mathrm{C}$ for $I T S, 58^{\circ} \mathrm{C}$ for $S S U$, and $60^{\circ} \mathrm{C}$ for $T U B$, and $70 \mathrm{~s}$ extension at $72^{\circ} \mathrm{C}$, followed by a final extension of 10 $\min$ at $72^{\circ} \mathrm{C}$.
PCR products were visualized by staining the gel with $0.8 \%$ to $1 \%$ gelview, purified and cloned into pMD18-T vector followed by transformation into Escherichia coli DH5a. More than two clones derived from each PCR product were sequenced (Invitrogen by Life Technologies, Shanghai) using universal forward primer M13F and reverse primer M13R. Sequences obtained were assembled and edited by DNAman (Lynnon Biosoft, Quebec). To compare the identity among these isolates, a preliminary automatic alignment of ITS, SSU, and TUB amplified from the eight isolates was generated using DNAman. In addition, the sequences of $L S U$, $I T S, S S U$, and $T U B$ were compared with the sequences downloaded from the National Centre for Biotechnology Information (NCBI). Datasets from each of the four regions were aligned using the ClustalX 1.81 (22). Phylogenetic analysis was performed using Mega 4.0 (20) with neighbor-joining method and Kimura 2-parameter model. Gaps were treated as missing data. All positions containing gaps and missing data were eliminated from the dataset. Clade stability of the trees resulting from the parsimony analyses was assessed by bootstrap with 1,000 replicates.

\section{Results}

Symptoms and isolation of causal agent. Leaf spots were initially small, needle-like, and reddish to brown. They enlarged gradually to become circular or oval spots, 1 to $13 \mathrm{~mm}$ in diameter (Fig. 1A). On the lower surface, the lesion was often surrounded by an oily halo (Fig. 1C). Later, the spots paled gradually from the

Table 2. Primers and their sequences used in this study

\begin{tabular}{lllc}
\hline Locus & Primer & Primer DNA sequence $\left(\mathbf{5}^{\prime} \mathbf{-} \mathbf{3}^{\prime}\right)$ & Product size (bp) \\
\hline ITS & UN-UP18S-42 & CGTAACAAGGTTTCCGTAGGTGA & 500 \\
& UN-LO28S-22 & GTTTCTTTCCTCCGCTTATTGATAT & $1,300-1,400$ \\
LSU & LR0R & ACCCGCTGAACTTAAGC & \multirow{2}{*}{$500-600$} \\
LSU & NMS1 & ATCCTGAGGGAAACTTC & 600 \\
& NMS2 & CAGCAGTGAGGAATATTGGTCAATG & \\
& Bt-T2m-Up & GCGGATCATCGAATTAAATAACAT & \\
& Bt-LEV-Lo1 & CAACTGGGCTAAGGGTCATT & \\
\hline
\end{tabular}
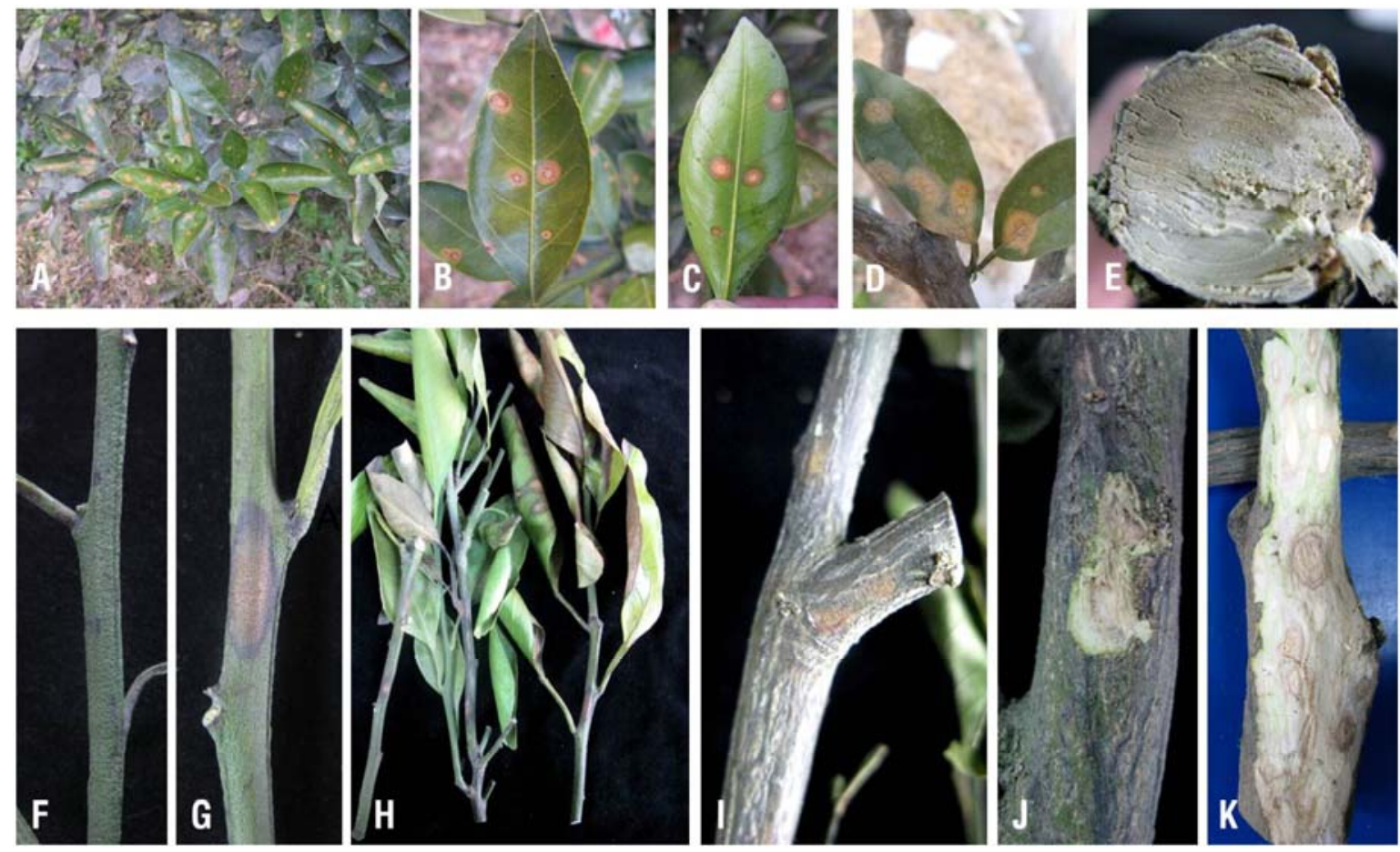

Fig. 1. Symptoms of Citrus target spot caused by Cryptosporiopsis citricarpa sp. nov. A, view of infected shoots; B, symptoms on upper surface of the leaf; C, symptoms on lower surface of the leaf; $\mathbf{D}$, symptoms on leaves of kumquat (Fortunella margarita); $\mathbf{E}$, cross section of infected branch; $\mathbf{F}$, initial symptom on shoots; $\mathbf{G}$, enlarged lesion on shoots; $\mathbf{H}$, symptoms on leaves and shoots; I-J, symptom on bark of branch; J-K, symptoms on wood of infected branches. 
center, black dots (acervuli) were produced in a circular arrangement on the upper surface of the lesions (Fig. 1B and D) and gained the typical appearance of "target spots". Target spots were often scattered on the leaves, but merging of two to three lesions was frequently observed. The symptoms on kumquat were similar to those on Satsuma mandarin (Fig. 1D).

The causal organism was also found to be associated with diseased petioles, shoots, branches, and standing trunks of citrus. The lesions on shoots were similar to those on leaves, initially very small, reddish to brown, slightly sunken spots (Fig. 1F); they then enlarged and became circular, elliptical, or spindle-shaped. These spots gradually became gray-brown at the center with dark brown margins (Fig. $1 \mathrm{G}$ and $\mathrm{H}$ ). Once the branches were infected, the bark became red-brown to dark brown (Fig. 1I and J), and the wood became red-brown to gray-brown (Fig. 1E, J, and K). Heavily infected shoots or branches were eventually blighted when the lesions girded. Seriously infected trees died. However, the disease was not observed on fruits either in orchards or in postharvest.

Prevalence and disease incidence. Target spot of citrus was first observed by local extension staffs in the winter of 2006 in Chenggu County, China, and gradually became more serious. The disease usually appeared around the middle of December. The incidence then increased and peaked in February. Infected leaves started to defoliate in March. Heavy defoliation occurred in April, and many infected trees became leafless and died in April. Interestingly, freshly sprouting leaves in May did not show any symptoms until the following winter.

This disease was present in all 10 orchards investigated, where Satsuma mandarin and kumquat were both grown. Both seedlings and fruit-bearing trees were susceptible. In comparison, the disease was more serious in orchards located in lowland fields than in those located on hillsides. Seedlings mulched with rice straw were less seriously infected than those without any mulching.

Isolation of causal fungi. In 2010, 3 and 8 isolates of Cryptosporiopsis sp. were isolated from 6 infected leaves and 11 branches, respectively. In 2011, 19, 6, and 10 isolates of Cryptosporiopsis sp. were isolated from 21 infected leaves, 6 infected shoots, and 13 infected branches, respectively. Strains SXL1, SXL6, and SXL10 were selected as representatives for Cryptosporiopsis sp. in pathogenicity tests.

Pathogenicity tests. Satsuma mandarin leaves inoculated with strains SXL1, SXL6, and SXL10 (C. citricarpa) on 27 May 2010 did not show any symptoms until December 2010. Distinct disease symptoms were observed on 22 February 2011. For strain SXL6, 18 out of 25 leaves $(72 \%)$ inoculated in May, and all 25 leaves $(100 \%)$ inoculated in December showed obvious symptoms that were similar to those in orchards. Black dots (acervuli) were observed on the lesions. The morphological characteristics of acervuli, conidiophores, and conidia were identical to those observed on naturally infected leaves collected in orchards. The same fungus was reisolated from 10 inoculated leaves $(100 \%)$, thus fulfilling Koch's postulates. The results for stains SXL1 and SXL10 gave similar results, thus the data were not given.

Very small dark brown necrotic spots ( 2 to $4 \mathrm{~mm}$ diameter) were seen on the inoculated rinds of fruits at 14 days. The spots were hard, resembling a healed wound. The above small dark brown necrotic spots did not enlarge, even though transferred to inoculated fruits at $4^{\circ} \mathrm{C}$. Fungal cultures could not be reisolated from the spots on the inoculated fruits.

Identification by the $L S U, I T S, S S U$, and $T U B$ sequence alignment. Sequence alignments indicated that the ITS of the eight selected isolates (Table 1) had $99.90 \%$ identity, the SSU and TUB of these eight isolates had 99.73 and $99.86 \%$ identity, respectively, indicating that they belonged to the same species.

The sequence of $L S U$ amplified from representative strain SXL6 was used to BLAST-search the GenBank database. The $L S U$ dataset for strain SXL6 had high identity to that of Neofabrea malicorticis (AY544662), N. alba (AY064705), and Cryptosporiopsis californiae (GU973597). Phylogenetic analysis of the $L S U$ regions from these species and several other pathogenic species in Helotiales downloaded from GenBank resulted in a phylogram comprising seven clades. SXL6 clustered with species of Neofabraea, Cryptosporiopsis, and Pezicula in Dermateaceae (Fig. 2).

The ITS sequence of strain SXL6 shared high identity with its homologues in Neofabraea spp. and Cryptosporiopsis spp., such as N. perennans (AF281389, 98\%), N. malicorticis (AF281383, 97\%), N. alba (AF141190, 97\%), C. actinidiae (EU482301, 97\%),

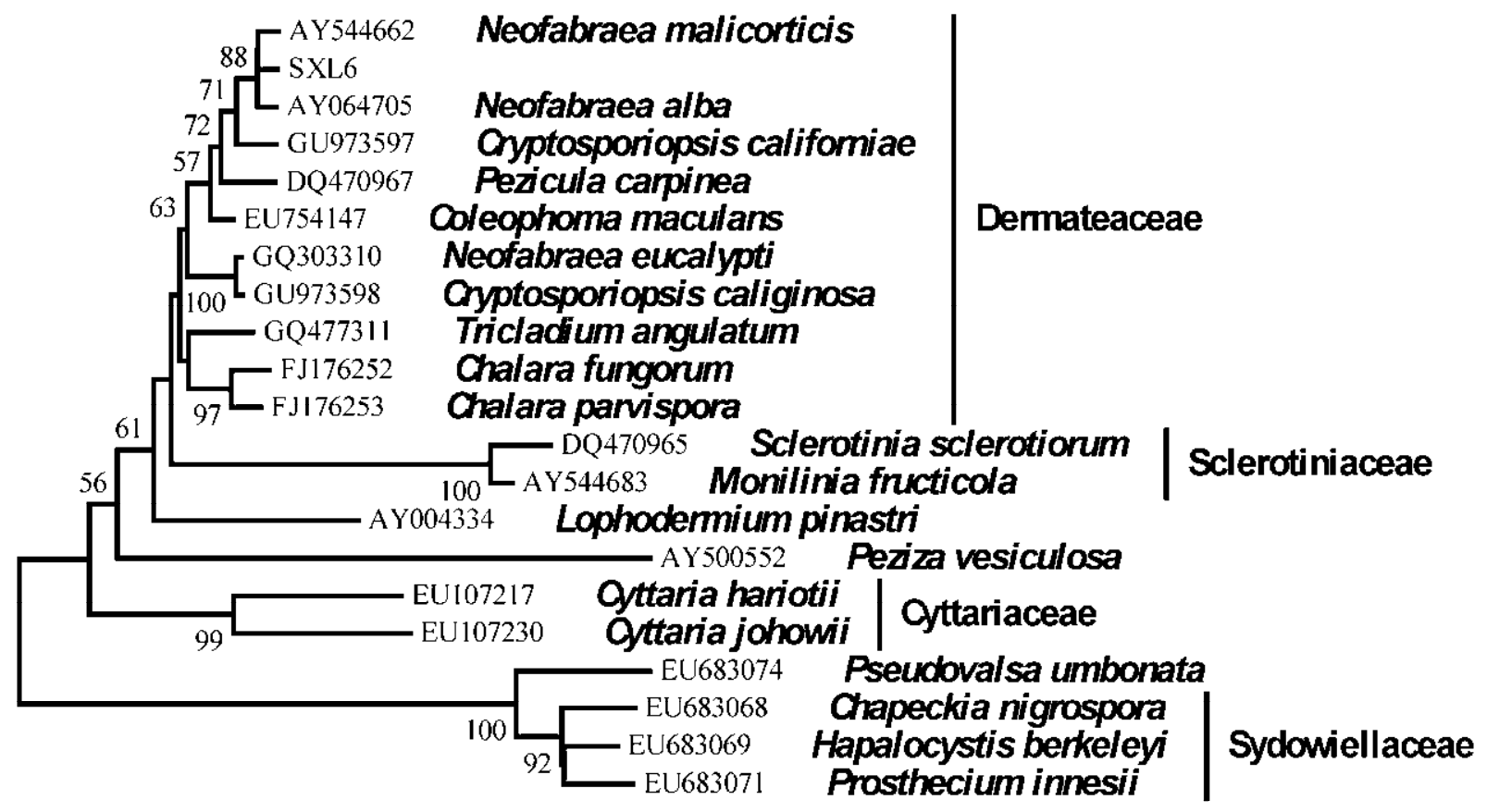

$\stackrel{\longmapsto}{0.01}$

Fig. 2. Phylogenetic relationships among isolates of Neofabraea spp., Cryptosporiopsis spp., and Cryptosporiopsis-like species generated by N-J analysis of LSU sequences using Kimura 2-parameter model. Bootstrap support values higher than 50\% from 1,000 replicates are shown at the nodes. 
and P. sporulosa (AF141172, 89\%). The SSU of SXL6 was $96 \%$ identical to that of $N$. krawtzewii (AF281431), and TUB was $91 \%$ identical to that of $N$. perennans (AF281474). Phylogenetic analysis of the ITS regions from these eight isolates (SXL1 to SXL6, SXL10 and SXL11) and 20 GenBank sequences representing known species in Neofabraea and Cryptosporiopsis indicated that the eight isolates derived from citrus target spots formed a monophyletic group with $99 \%$ bootstrap support (Fig. 3). They constituted an independent clade which was distinct from strains of $N$. perennans, $N$. malicorticis, and $N$. alba (Fig. 3). The phylograms inferred from $S S U$ and $T U B$ showed similar results to that from ITS (data not shown).
Comparison of morphology. Morphological comparison indicated that the taxon isolated from citrus target spot was morphologically distinct from other Cryptosporiopsis species (Table 3 ). In general, the strain produced both macroconidia and microconidia that were different from those of other species (Table 3).

Based on morphological and molecular evidence, we concluded that the causal agent of citrus target spot in Chenggu County, China belongs to an undescribed species within Cryptosporiopsis (or Neofabarea). Considering the teleomorph of this pathogen has not been found, the anamorphic name Cryptosporiopsis citricarpa is assigned to accommodate it.

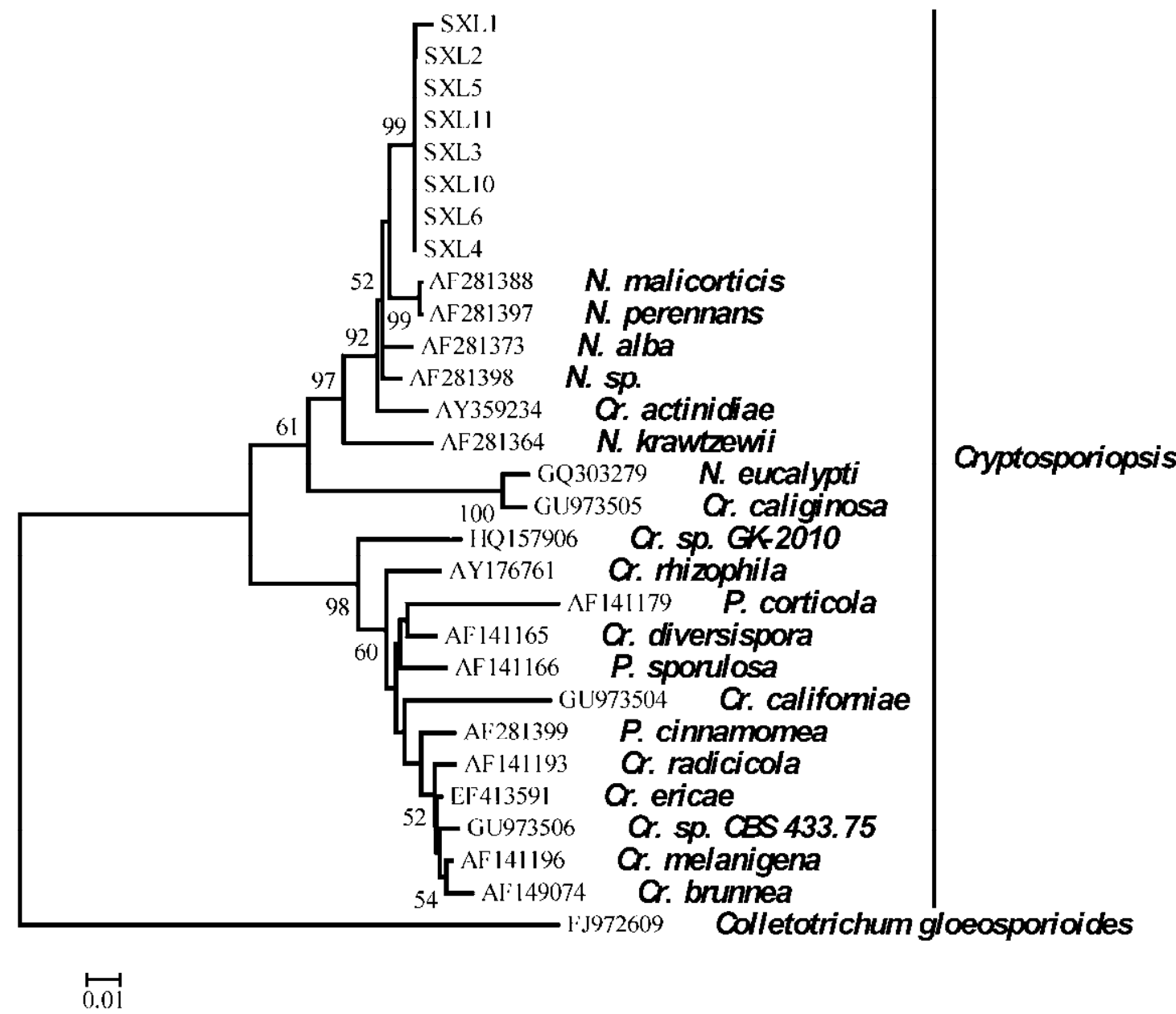

Fig. 3. Phylogenetic relationships among strains of Neofabraea spp. and Cryptosporiopsis spp. generated by N-J analysis of internal transcribed spacer rDNA sequences using Kimura 2-parameter model. Cr. $=$ Cryptosporiopsis, $N$. =Neofabraea, $P .=$ Pezicula. Bootstrap support values higher than $50 \%$ from 1,000 replicates are shown at the nodes. The tree is rooted with Colletotrichum gloeosporioides.

Table 3. Dimensions of conidia and conidiophores of related species of Neofabraea and Cryptosporiopsis

\begin{tabular}{|c|c|c|c|}
\hline Species & Macroconidia $(\mu \mathrm{m})$ & Microconidia $(\mu \mathrm{m})$ & $\begin{array}{c}\text { Conidiophores } \\
\text { or conidiogenous cells }(\mu \mathrm{m})\end{array}$ \\
\hline C. citricarpa & $21-40 \times 5-9^{a}$ & $5-10 \times 2-4^{b}$ & $12-49 \times 4-7^{a}$ \\
\hline N. malicorticis (25) & $15-35 \times 3-6^{c}$ & $5-8 \times 1-1.5^{\mathrm{c}}$ & \\
\hline N. perennans $(25)$ & $12-25 \times 3-6^{d}$ & $6-10 \times 1.5-3^{d}$ & \\
\hline N. alba (25) & $17-30 \times 2.5-3.5^{\mathrm{a}}$ & $15-18 \times 0.5-1^{\mathrm{a}}$ & \\
\hline C. kienholzii (17) & $12-17.5 \times 2.5-3.5^{\mathrm{c}}$ & $2.5-6.5 \times 1.5-2.5^{\mathrm{c}}$ & \\
\hline N. krawtzewii (21) & $25-45 \times 4.5-5^{\mathrm{a}}$ & $4.5-8 \times 1.5-2.5^{\mathrm{a}}$ & $25-35 \times 4^{\mathrm{a}}$ \\
\hline C. citri (14) & Not related & $9-11.5 \times 4-5.5^{\mathrm{a}}$ & $9.5-12 \times 2.5-5^{\mathrm{a}}$ \\
\hline C. californiae (5) & Not related & $15-18 \times 4.5-5.2^{\mathrm{e}}$ & $8-11 \times 2.5-3.5^{\mathrm{e}}$ \\
\hline
\end{tabular}

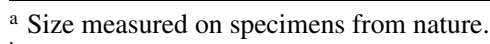

${ }^{\mathrm{b}}$ Size measured on specimens in culture on potato dextrose agar.

${ }^{\mathrm{c}}$ Size measured on specimens in culture on oatmeal agar.

${ }^{\mathrm{d}}$ Size measured from drawings based on specimens from nature.

e Size measured on specimens in culture. 


\section{Taxonomic description}

Cryptosporiopsis citricarpa L. Zhu, H.Y. Li \& K.D. Hyde, sp. nov. Figs. 4, 5, and 6.

MycoBank: 563094.

Description from leaves. Conidiomata (Fig. 4A) epigenous, stromatic, acervular, raised, subcuticular to epidermal, 210 (111-413) $\mu \mathrm{m}$ in diameter, mostly found on the upper surface of leaves, separate or confluent, dark brown. Conidiomata composed of interwoven, narrow, slightly brown hyphae. Setae absent. Conidiophores (Fig. 4B and C) aseptate or occasionally with one septum, unbranched, hyaline, 12-49 × 4-7 $\mu \mathrm{m}(n=50)$. Conidia (Fig. 4D), 21-40 $\times 5-9 \mu \mathrm{m}$ $(n=50)$, borne singly at the tips of the conidiophores, cylindricfusiform, sometimes weakly to strongly curved, apex rounded to slightly pointed, base more or less conical then truncate, unicellular or occasionally 1-septate, hyaline, contents granular.
Description from culture. Acervuli not formed in culture. The conidiogenous cells were not different from hyphae. Conidia produced readily within 7 to 10 days following incubation at $20^{\circ} \mathrm{C}, 5-$ $10 \times 2-4 \mu \mathrm{m}(n=50)$, much smaller than those produced on leaves, thus assigned as microconidia (Fig. 4I). The microconidia were ellipsoidal, oblong-ellipsoidal, slightly asymmetrical to curved, base more or less conical then truncate, hyaline, with abundant guttules (Fig. 4J). Microconidia germinated in a similar way as that of macroconidia (Fig. 4K and L). Chlamydospores and setae were not observed in culture, whereas sclerotia formed at the bottom (Fig. 5A and B) or surface (Fig. 5C) of cultures after 2 months of incubation.

Material examined. CHINA, Shaanxi, Chenggu, on living leaf of Satsuma mandarin (Citrus unshiu Marcov.) (Rutaceae) as leaf target spot, 27 March 2010, L. Zhu, CBS H-20689 (holotype); ex-
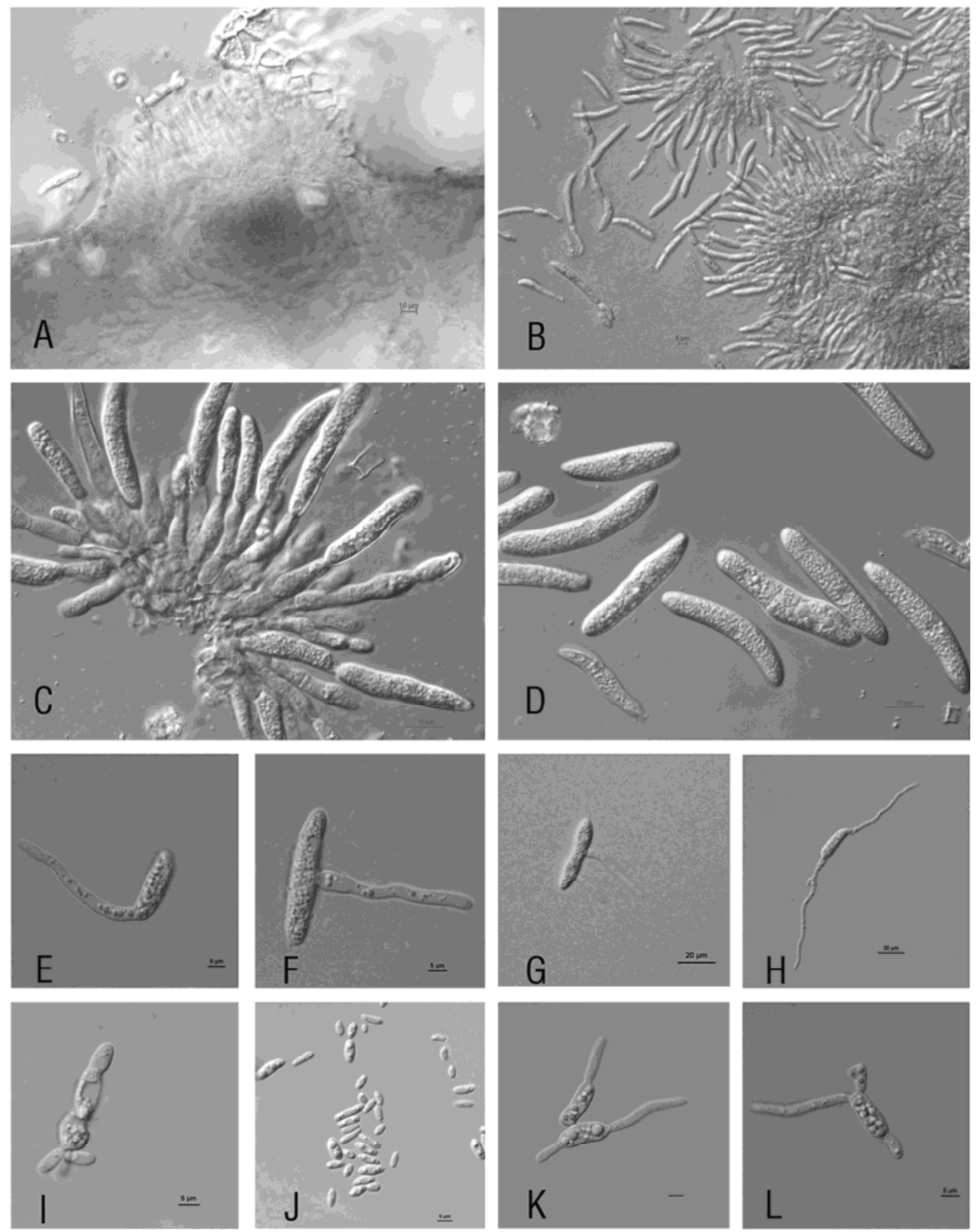

Fig. 4. Morphological characters of Cryptosporiopsis citricarpa. A, Acervulus formed leaves; B and C, conidiophores with macroconidia formed on leaves; D, macroconidia formed on leaves; $\mathbf{E}, \mathbf{F}, \mathbf{G}$, and $\mathbf{H}$, macroconidia germinated in sterile distilled water; I, macroconidia formed oval to elliptical microconidia budding off from the side of the spores; J, microconidia raised in culture; $\mathbf{K}$ and $\mathrm{L}$, microconidia germinated in sterile distilled water. B, E, F, I, J, K, and L, scale bars $=5 \mu \mathrm{m} ; \mathbf{A}, \mathbf{C}$, and $\mathbf{D}, \mathrm{scale}$ bars $=10$ $\mu \mathrm{m} ; \mathbf{G}$ and $\mathbf{H}$, scale bars $=20 \mu \mathrm{m}$. 
type culture ZJUCCSXL6 = CBS 130297 = CGMCC 3.14880; Chenggu Wulang, on leaf of Satsuma mandarin with leaf target spot, 22 February 2011, L. Zhu, ex-type culture ZJUCCSXL2 = CBS 130531 = CGMCC3.14876; Chenggu Wulang, on shoot of Satsuma mandarin with leaf target spot, 22 February 2011, L. Zhu, ex-type culture ZJUCCSXL3 = CBS 130532 = CGMCC3.14877; Chenggu, Dongzha nursery garden, on leaf of Satsuma mandarin with leaf target spot, 22 February 2011, L. Zhu, ex-type culture ZJUCCSXL4 = CBS 130533 = CGMCC3.14878; Chenggu Chencun, on leaf of Satsuma mandarin with leaf target spot, 22 February 2011, L. Zhu, ex-type culture ZJUCCSXL5 = CBS $130534=$ CGMCC3.14879; Chenggu Yaozhuang, on leaf of Satsuma mandarin with leaf target spot, 27 March 2010, L. Zhu, ex-type culture ZJUCCSXL1 = CBS $130530=$ CGMCC3.14875; Chenggu, Dongzha nursery garden, on leaf of Fortunella margarita with leaf target spot, 27 March 2010, L. Zhu, ex-type culture ZJUCCSXL10 $=$ CBS 130535 = CGMCC3.14882; Chenggu Guanwangbao, on leaf of Satsuma mandarin with leaf target spot, 7 March 2011, L. Zhu, ex-type culture ZJUCCSXL11 = CBS $130536=$ CGMCC3.14883.

Notes: Conidia forming on leaves were larger than those formed in culture, and the former have been termed as "macroconidia", the latter termed as "microconidia". Conidia germinated in sterile distilled water after 6 to $7 \mathrm{~h}$ at $20^{\circ} \mathrm{C}$. Germ tubes were produced from the ends or sides of the conidia (Fig. 4E to H). Sometimes, instead of producing germ tubes, the macroconidia formed oval to elliptical, one-celled hyaline spores (Fig. 4I), resembling microconidia produced in culture.

Culture characteristics. The three selected isolates (SXL1, SXL6, and SXL10; Table 1) produced the same type of colony on PDA, OA, and MEA; thus the data from isolate SXL6 (derived from holotype) is presented here. After 14 days of incubation at $20^{\circ} \mathrm{C}$, colonies reached 28 to $31 \mathrm{~mm}, 25$ to $32 \mathrm{~mm}$, and 19 to 24 $\mathrm{mm}$ in diameter on PDA, OA, and MEA, respectively. On PDA, the colony was rather dense, initially white to gray, and later became dark blue or black, the aerial mycelia was copious, white, and cottony (Fig. 6A). The reverse side of the colony was milk-yellowish, interdispersed with blackish blue circles (Fig. 6D). On OA, the colony was dense, initially gray to light brown, then becoming dark blue and black, with copious white and feathery aerial mycelia (Fig. 6B). The reverse side of the colony was initially white to grayish, becoming light orange, brown and dark brown, or gradually black (Fig. 6E). On MEA, the texture of the colony was dense, at first whitish, gradually becoming brownish to black, with copious, white and feathery or loose cottony aerial mycelia (Fig. 6C). The reverse side of the colony was whitish to grayish, gradually becoming brown to black (Fig. 6F). The margins of colonies grown on these media were smooth to entire, or with a
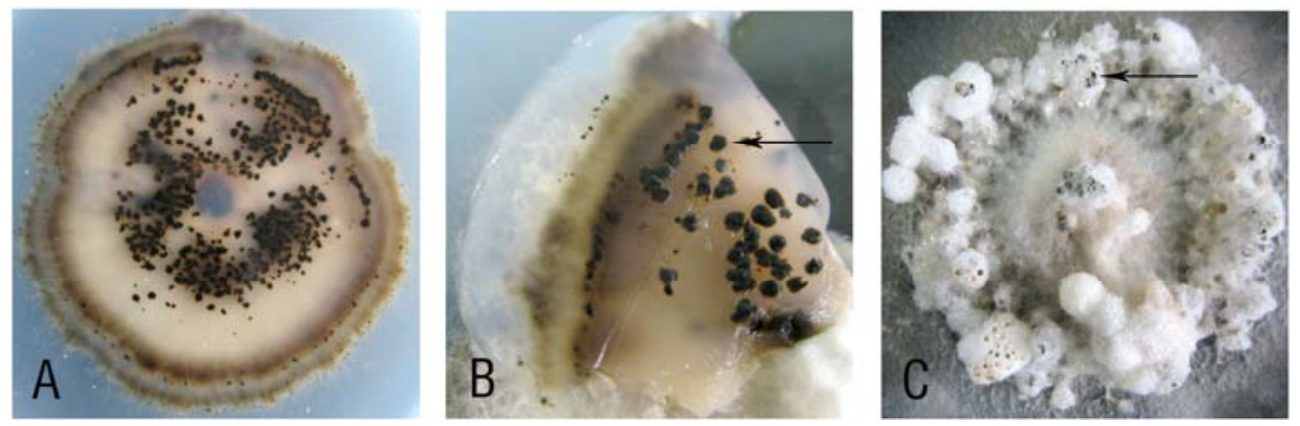

Fig. 5. Sclerotia formed on surface or bottom of culture within a 2-month incubation period. A and B, Lower view of plate; C, upper view of plate.
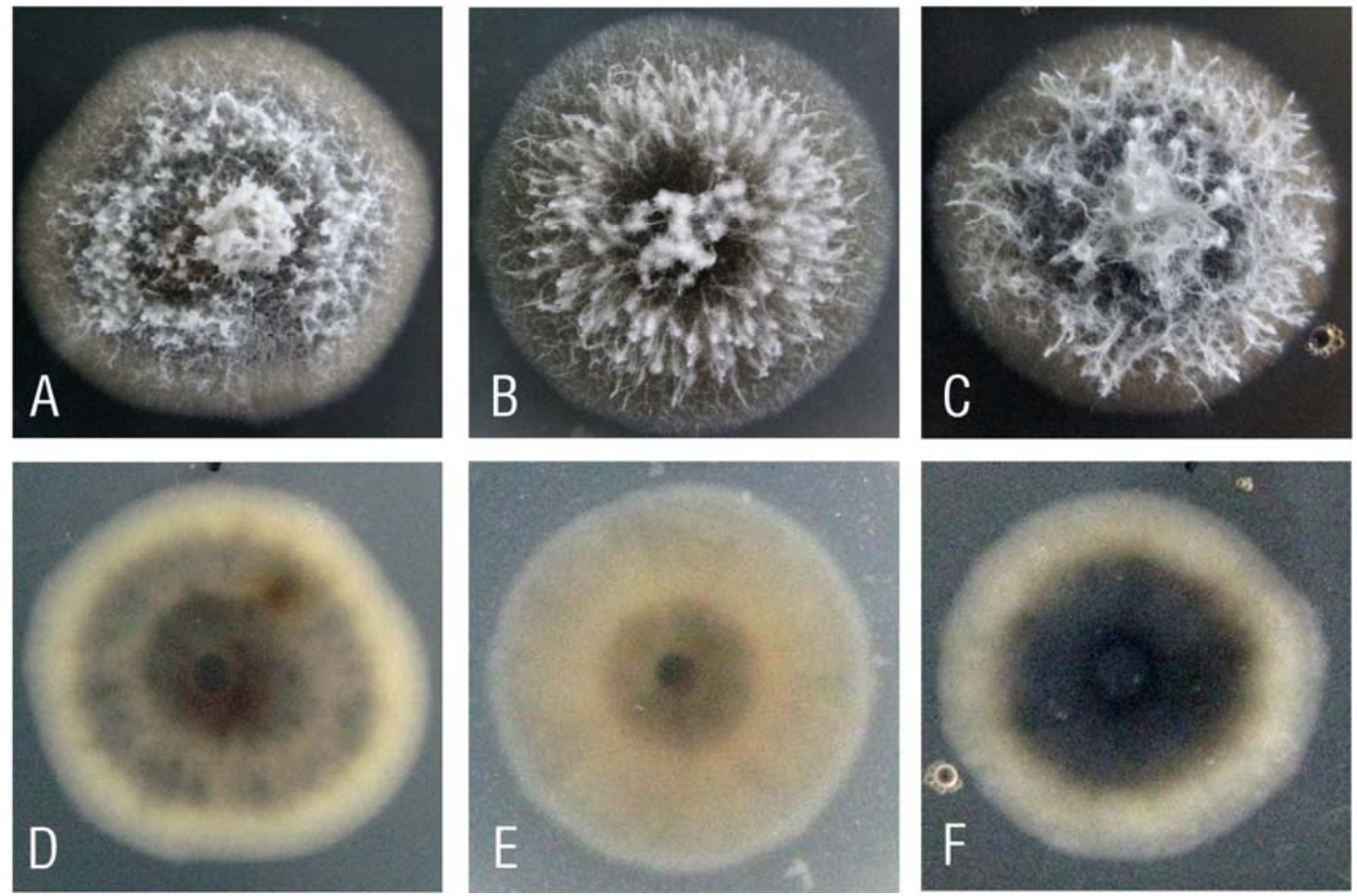

Fig. 6. Cultural characters of Cryptosporiopsis citricarpa on different media at 14 days of incubation at $20^{\circ} \mathrm{C}$. A, B, and C, Upper view of plates; D, E, and F, lower view of plates; $\mathbf{A}$ and $\mathbf{D}$, on potato dextrose agar; $\mathbf{B}$ and $\mathrm{E}$, on oatmeal agar; $\mathbf{C}$ and $\mathrm{F}$, on malt extract agar. 
low fringe of white radiating hyphae (Fig. 6A to C), diffusing pigments were usually absent (Fig. $6 \mathrm{D}$ to $\mathrm{F}$ ).

Temperature response. The temperature response of the three tested isolates was similar. Temperature range for their growth was between 0 and $28^{\circ} \mathrm{C}$ with an optimum at $20^{\circ} \mathrm{C}$ (Fig. 7). At the optimum temperature, the mean growth rate was $1.8 \mathrm{~mm}$ per day on PDA. At $30^{\circ} \mathrm{C}$, growth of $C$. citricarpa ceased but the isolates remained viable, since growth could be resumed when switching the plates back to $20^{\circ} \mathrm{C}$. However, the isolates were killed after incubation for 14 days at $35^{\circ} \mathrm{C}$, as their growth did not recover after switching the plates to $20^{\circ} \mathrm{C}$. The temperature response of $\mathrm{C}$. citricarpa was similar to CLX4144, the reference isolate of $C$. kienholzii, included in this study.

C. citricarpa produced abundant conidia in culture between 4 and $20^{\circ} \mathrm{C}$, but only a few at $25^{\circ} \mathrm{C}$. No conidia were produced at $0^{\circ} \mathrm{C}$ or at $28^{\circ} \mathrm{C}$ or above. The texture of colony and shape of microconidia were affected by the incubation temperatures. At 0 and $4^{\circ} \mathrm{C}$, the colony was much looser, lighter in color, and lacked or comprised sparse aerial mycelium than at other temperatures; the conidia produced at $4^{\circ} \mathrm{C}$ were distinctly curved.

\section{Discussion}

Target spot of Citrus has become an important disease in Shaanxi Province, China, seriously affecting production. A high proportion of citrus trees in Chenggu County were diseased or dead, and several orchards have been destroyed. No effective control strategy has yet been developed. Fortunately, in surveys of citrus fungal diseases across China over the past 3 years, this disease has not been found in any other citrus-producing regions, and this fungus did not infect citrus fruits.

The temperature range for $C$. citricarpa growth in vitro was 0 to $28^{\circ} \mathrm{C}$, with an optimum at $20^{\circ} \mathrm{C}$ (Fig. 7). Together with the fact that the disease prevailed in late winter and early spring, disappeared in the summer and autumn, and leaves inoculated in either May or December produced symptoms in the following February, this suggests that $C$. citricarpa is a low-temperature-adapted pathogen. The temperature response of C. citricarpa is similar to Neofabraea spp. causing Bull's-eye rot of apple and pear, and N. krawtzewii causing canker disease of poplars (21).

Chenggu (107.3E, 33.2N) is located in the hinterland of Hanzhong Basin of southern Shaanxi. Qinling Mountains, located to the north, block the cold air blowing down from Siberia, which allows Chenggu to be the most northern citrus-producing region of China. The temperatures in Chenggu are lower than in other citrusproducing areas in China, having an annual mean of $14.2^{\circ} \mathrm{C}$ in Chenggu versus $17^{\circ} \mathrm{C}$ in Guangyuan (105E, 32N), Sichuan. The main variety of citrus grown in Chenggu is Satsuma mandarin, which is the most common variety of citrus grown in China. Thus, we suggest that the outbreak of citrus target spot in Chenggu is probably related to the location, due to the low temperatures in this region. However, this study demonstrated that $C$. citricarpa could

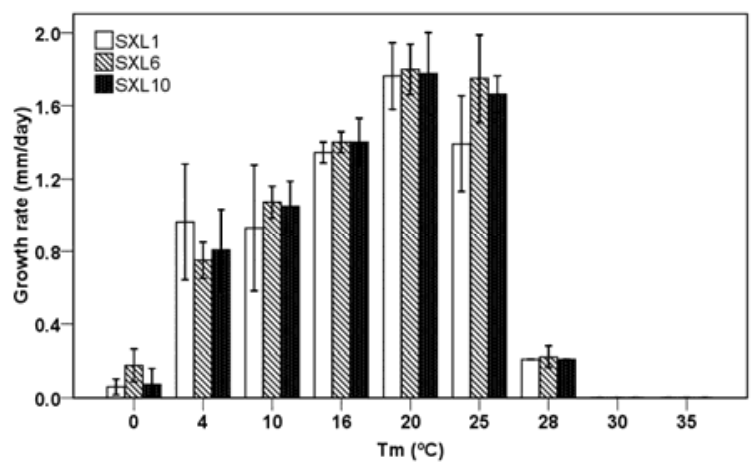

Fig. 7. Growth rate of three isolates (SXL1, SXL6, and SXL10) at different temperatures. Colony diameter was measured after 14 days of incubation. Columns represented means of three replicates, and bars represent standard deviation. The experiment was conducted twice. also cause disease in Hangzhou (120.2E, 30.3N) when leaves were artificially inoculated. The potential risk of the occurrence of this disease in subtropical or tropical regions therefore should be investigated.

Cryptosporiopsis citri was identified as a leaf spotting disease of older leaves of several Citrus species in Pacific Island countries including the Cook Islands, Fiji, Niue, Tonga, Vanuatu, and Western Samoa (12,14). The symptoms (based on publications) induced by $C$. citri were somewhat similar to those described in this study. However, the size and shape of conidia in C. citri were distinctly different from $C$. citricarpa (Table 3 ). Only one type of conidia was reported for $C$. citri (12), and these were smaller than the macroconidia, but larger than the microconidia of $C$. citricarpa (Table 3). Because the sequence information for $C$. citri is lacking in GenBank, we could not compare these fungal pathogens of citrus at the DNA sequence level. However, we treat $C$. citricarpa as a new species because it is morphologically and physiologically different: $C$. citricarpa prefers cold regions with an optimum growth at $20^{\circ} \mathrm{C}$, while although the optimum growth temperature for $C$. citri was not recorded, it is likely to be higher as this taxon is from tropical regions. Based on our best knowledge, target spot is a new destructive disease of citrus. Clarification of the causal agent of this disease will help us develop strategies to control this disease, and alert us to its spread and new outbreaks.

\section{Acknowledgments}

We gratefully acknowledge Changlin Xiao (Washington State University, WA) for providing us with Neofabraea isolates from apples and pears for reference and Xinshun Ding (The Samuel Roberts Noble Foundation) for critical English editing. This work was granted by the National Foundation of Natural Science of China (31071649) and the earmarked fund for Modern Agro-industry Technology Research System (MATRS). The Global Research Network for Fungal Biology and King Saud University are also thanked for support.

\section{Literature Cited}

1. Abeln, E. C. A., de Pagter, M. A., and Verkley, G. J. M. 2000. Phylogeny of Pezicula, Dermea and Neofabraea inferred from partial sequences of the nuclear ribosomal RNA gene cluster. Mycologia 92:685-693.

2. Bakkeren, G., Kronstad, J. W., and Lévesque, C. A. 2000. Comparison of AFLP fingerprints and ITS sequences as phylogenetic markers in Ustilaginomycetes. Mycologia 92:510-521.

3. Cai, L., Hyde, K. D., Taylor, P. W. J., Weir, B., Waller, J., Abang, M. M., Zhang, J. Z., Yang, Y. L., Phoulivong, S., Liu, Z. Y., Prihastuti, H., Shivas, R. G., McKenzie, E. H. C., and Johnston, P. R. 2009. A polyphasic approach for studying Colletotrichum. Fungal Divers. 39:183-204.

4. Cheewangkoon, R., Groenewald, J. Z., Summerell, B. A., Hyde, K. D., Toanun, C., and Crous, P. W. 2009. Myrtaceae, a cache of fungal biodiversity. Persoonia 23:55-85.

5. Cheewangkoon, R., Groenewald, J. Z., Verkley, G. J. M., Hyde, K. D., Wingfield, M. J., Gryzenhout, M., Summerell, B. A., Denman, S., Toanun, C., and Crous, P. W. 2010. Re-evaluation of Cryptosporiopsis eucalypti and Cryptosporiopsis-like species occurring on Eucalyptus leaves. Fungal Divers. 44:89-105.

6. de Jong, S. N., Lévesque, C. A., Verkley, G. J. M., Abeln, E. C. A., Rahe, J. E., and Braun, P. G. 2001. Phylogenetic relationships among Neofabraea species causing tree cankers and bull's-eye rot of apple based on DNA sequencing of ITS nuclear rDNA, mitochondrial rDNA, and the $\beta$-tubulin gene. Mycol. Res. 105:658-669.

7. Dugan, F. M. 1993. Anthracnose, perennial canker and bull's-eye rot of apple and pear. Tree Fruit Postharv. J. (USA) 4:5-6.

8. Gariepy, T. D., Rahe, J. E., Lévesque, C. A., Spotts, R. A., Sugar, D. L., and Henriquez, J. L. 2005. Neofabraea species associated with bull's-eye rot and cankers of apple and pear in the Pacific Northwest. Can. J. Plant Pathol. 27:118-124.

9. Goh, T. K. 1999. Single-spore isolation using a hand-made glass needle. Fungal Divers. 2:47-63.

10. Henriquez, J. L., Sugar, D., and Spotts, R. A. 2004. Etiology of bull's eye rot of pear caused by Neofabraea spp. in Oregon, Washington, and California. Plant Dis. 88:1134-1138.

11. Jackson, H. S. 1913. Apple tree anthracnose; a preliminary report. Oregon State Agric. Exp. Stn. Bienn. Crop Pest Hortic. Rep. 1911-1912:178-197.

12. Johnston, P. R., and Fullerton, R. A. 1988. Cryptosporiopsis citri sp. nov.; cause of a Citrus leaf spot in the Pacific Islands. N.Z. J. Exp. Agric. 16:159163.

13. Li, K.-N., Rouse, D. I., and German, T. L. 1994. PCR primers that allow intergeneric differentiation of ascomycetes and their application to Verticillium spp. Appl. Environ. Microbiol. 60:4324-4331.

14. Ray, J. D., McTaggart, A. R., and Shivas, R. G. 2008. First record of Cryptosporiopsis citri on lime in Australia. Australas. Plant Dis. Notes 
$3: 158-159$

15. Sankaran, K. V., Sutton, B. C., and Balasundaran, M. 1995. Cryptosporiopsis eucalypti sp. nov., causing leaf spots of eucalypts in Australia, India and USA. Mycol. Res. 99:827-830.

16. Schulz, B., Boyle, C., Draeger, S., Römmert, A. K., and Krohn, K. 2002. Endophytic fungi: A source of novel biologically active secondary metabolites. Mycol. Res. 106:996-1004.

17. Spotts, R. A., Seifert, K. A., Wallis, K. M., Sugar, D., Xiao, C. L., Serdani, M., and Henriquez, J. L. 2009. Description of Cryptosporiopsis kienholzii and species profiles of Neofabraea in major pome fruit growing districts in the Pacific Northwest USA. Mycol. Res. 113:1301-1311.

18. Strobel, G. A., Miller, R., Vincent, M.-M. C., Condron, M. M., Teplow, D. B., and Hess, W. M. 1999. Cryptocandin, a potent antimycotic from the endophytic fungus Cryptosporiopsis cf. quercina. Microbiology 145:1919.

19. Sutton, B. C. 1980. The Coelomycetes: Fungi imperfecti with pycnidia, acervuli and stromata. Commonwealth Mycological Institute, Kew, Surrey, UK.

20. Tamura, K., Dudley, J., Nei, M., and Kumar, S. 2007. MEGA4: Molecular Evolutionary Genetics Analysis (MEGA) software ver. 4.0. Mol. Biol. Evol. 24:1596-1599.

21. Thompson, G. E. 1939. A canker disease of poplars caused a new species of Neofabraea. Mycologia 31:455-465.
22. Thompson, J. D., Gibson, T. J., and Higgins, D. G. 2002. Multiple sequence alignment using ClustalW and ClustalX. Curr. Protocols Bioinform. Ch. 2: Unit 2.3.

23. Timmer, L. W., Garnsey, S. M., and Graham, J. H. 2000. Compendium of Citrus Diseases. 2nd ed. American Phytopathological Society, St. Paul, MN.

24. van Burik, J.-A. H., Schreckhise, R. W., White, T. C., Bowden, R. A., and Myerson, D. 1998. Comparison of six extraction techniques for isolation of DNA from filamentous fungi. Med. Mycol. 36:299-303.

25. Verkley, G. J. M. 1999. A monograph of the genus Pezicula and its anamorphs. Stud. Mycol. 44:1-180.

26. Vilgalys, R., and Hester, M. 1990. Rapid genetic identification and mapping of enzymatically amplified ribosomal DNA from several Cryptococcus species. J. Bacteriol. 172:4238-4246.

27. Wulandari, N. F., To-anun, C., Hyde, K. D., Duong, L. M., de Gruyter, J., Meffert, J. P., Groenewald, J. Z., and Crous, P. W. 2009. Phyllosticta citriasiana sp. nov., the cause of Citrus tan spot of Citrus maxima in Asia. Fungal Divers. 34:23-39.

28. Xiao, C. L., and Rogers, J. D. 2004. A postharvest fruit rot in d'Anjou pears caused by Sphaeropsis pyriputrescens sp. nov. Plant Dis. 88:114-118.

29. Yang, Y. L., Liu, Z. Y., Cai, L., Hyde, K. D., Yu, Z. N., and McKenzie, E. H. C. 2009. Colletotrichum anthracnose of Amaryllidaceae. Fungal Divers. 39:123-146. 